\title{
Estudo da evapotranspiração máxima, mínima e anual sobre a bacia hidrográfica do rio Uruçuí Preto - Piaú, Brasil
}

Study of maximum, minimum and annual evapotranspiration on the watershed of rio Uruçuí Preto

- Piauí, Brazil

Estudio de evapotranspiración máxima, mínima y anual en la cuenca del río Uruçuí Preto - Piauí, Brasil

Raimundo Mainar de Medeiros ORCID: https://orcid.org/0000-0003-3455-9876 Universidade Federal Rural de Pernambuco, Brasil E-mail: mainarmedeiros@gmail.com Salatiel Ewen Braga

ORCID: https://orcid.org/0000-0002-3233-5361 Universidade Federal Rural de Pernambuco, Brasil E-mail: salatiel_braga@ hotmail.com

Manoel Vieira de França

ORCID: https://orcid.org/0000-0003-4973-9327 Universidade Federal Rural de Pernambuco, Brasil E-mail: manoelvieiraufrpe@gmail.com Wagner Rodolfo de Araújo ORCID: https://orcid.org/0000-0001-7203-0338 Universidade Estácio de Sá, Brasil E-mail: wagneraraujops@gmail.com

Luciano Marcelo Fallé Saboya ORCID: https://orcid.org/0000-0002-7586-6867 Universidade Federal de Campina Grande, Brasil E-mail: 1saboya@hotmail.com

Fernando Cartaxo Rolim Neto ORCID: https://orcid.org/0000-0002-6411-2058 Universidade Federal Rural de Pernambuco, Brasil

E-mail: fernandocartaxo@yahoo.com.br

Romildo Morant De Holanda ORCID: https://orcid.org/0000-0001-7945-3616 Universidade Federal Rural de Pernambuco, Brasil E-mail: romildomorantpesquisador@gmail.com

Márcia Liana Freire Pereira ORCID: https://orcid.org/0000-0002-3434-3349 Universidade Federal de Campina Grande, Brasil E-mail: liana.freire@gmail.com

\begin{abstract}
Resumo
Conhecer o clima local é um fator necessário para avaliação dos recursos hídricos, pois vários elementos meteorológicos são indispensáveis em todo ciclo hidrológico. Nesse contexto, os objetivos deste trabalho consistem em se estimar as evapotranspirações máxima, mínima e anual para a bacia hidrografica do rio Uruçuí Preto, estado do Piauí. A estimativa da evapotranspiração mínima, máxima e anual foi efetuada a partir das equações empíricas para a estimativa da temperatura do ar para a área da bacia em estudo. Foram utilizadas séries históricas de valores mensais da temperatura do ar, de períodos uniformizados com 30 anos de observações, para os 49 municípios que englobam a área de estudo. Com os dados dessas séries, foram ajustadas equações pelo método dos mínimos quadrados, obtendose equações de regressão linear múltipla e testaram o método estatístico. Com os dados de temperatura média do ar estimados, procedeu-se à estimativa da evapotranspiração pelo método empírico de Thornthwaite. Foram elaborados valores da temperatura média do ar, estimados pelo software Estima_T. A oscilação da evapotranspiração média da área estudada ocorre entre $92,4 \mathrm{~mm}$ em fevereiro a $170,1 \mathrm{~mm}$ no mês de outubro. A evapotranspiração máxima oscila entre 111,3 a 202,8 mm. A ETP mínima oscila entre 70,7 a 130,2 mm. As estimativas de ETP da bacia hidrográfica do rio Uruçuí Preto - Piauí apresentam dependência do posicionamento geográfico (latitude e longitude), e também da orografia local. Em termos médios, se sobressaem as classes de ocorrência de 70 a $125 \mathrm{~mm}, 125$ a $175 \mathrm{~mm}$ e a classe mais alta superior aos $200 \mathrm{~mm}$ que, juntas, totalizam 84,4\% da área de estudo, representando uma variação de 5,3 mm a 10,8 mm, em termos de evapotranspiração média diária, em praticamente toda a bacia.
\end{abstract}


Palavras-chave: Produção agrícola; Balanço hídrico; Demanda hídrica; Irrigação; Mudanças climáticas.

\begin{abstract}
Knowing the local climate is a necessary factor for assessing water resources, as several meteorological elements are indispensable throughout the hydrological cycle. In this context, the objectives of this work are to estimate the maximum, minimum and annual evapotranspirations for the hydrographic basin of the Uruçuí Preto River, state of Piauí. The estimate of the minimum, maximum and annual evapotranspiration was made from the empirical equations for the estimation of the air temperature for the area of the basin under study. Historical series of monthly air temperature values were used, from uniform periods with 30 years of observations, for the 49 municipalities that encompass the study area. With the data from these series, equations were adjusted by the least squares method, obtaining multiple linear regression equations and tested the statistical method. With the average air temperature data estimated, evapotranspiration was estimated using the empirical method of Thornthwaite. Values for the average air temperature were estimated, estimated by the Estima_T software. The average evapotranspiration oscillation in the studied area occurs between $92.4 \mathrm{~mm}$ in February and $170.1 \mathrm{~mm}$ in October. Maximum evapotranspiration ranges from 111.3 to $202.8 \mathrm{~mm}$. The minimum ETP ranges from 70.7 to $130.2 \mathrm{~mm}$. The ETP estimates of the Uruçuí Preto Piauí river basin are dependent on geographic positioning (latitude and longitude), and also on local orography. In average terms, the occurrence classes of 70 to $125 \mathrm{~mm}, 125$ to $175 \mathrm{~mm}$ and the highest class above $200 \mathrm{~mm}$ stand out, which together make up $84.4 \%$ of the study area, representing a variation of $5.3 \mathrm{~mm}$ to $10.8 \mathrm{~mm}$, in terms of average daily evapotranspiration, in practically the entire basin.
\end{abstract}

Keywords: Agricultural production; Hydric balance; Water balance; Water demand; Irrigation; Climate change.

\begin{abstract}
Resumen
Conocer el clima local es un factor necesario para evaluar los recursos hídricos, ya que varios elementos meteorológicos son indispensables en todo ciclo hidrológico. En este contexto, los objetivos de este trabajo son estimar las evapotranspiraciones máximas, mínimas y anuales para la cuenca hidrográfica del río Uruçuí Preto, estado de Piauí. La estimación de la evapotranspiración mínima, máxima y anual se realizó a partir de las ecuaciones empíricas para la estimación de la temperatura del aire para el área de la cuenca en estudio. Se utilizaron series históricas de valores de temperatura del aire mensuales, de períodos uniformes con 30 años de observaciones, para los 49 municipios que componen el área de estudio. Con los datos de estas series, las ecuaciones fueron ajustadas por el método de mínimos cuadrados, obteniendo ecuaciones de regresión lineal múltiple y probando el método estadístico. Con los datos de temperatura promedio del aire estimados, la evapotranspiración se estimó utilizando el método empírico de Thornthwaite. Se estimaron los valores de la temperatura media del aire, estimados por el software Estima_T. La oscilación de la evapotranspiración promedio en el área estudiada ocurre entre 92,4 $\mathrm{mm}$ en febrero y 170,1 mm en octubre. La evapotranspiración máxima varía de 111,3 a 202,8 mm. El ETP mínimo oscila entre 70,7 y 130,2 mm. Las estimaciones de ETP de la cuenca del río Uruçuí Preto - Piauí dependen del posicionamiento geográfico (latitud y longitud), y también de la orografía local. En términos promedio, se destacan las clases de ocurrencia de 70 a $125 \mathrm{~mm}, 125$ a $175 \mathrm{~mm}$ y la clase más alta por encima de $200 \mathrm{~mm}$, que en conjunto componen el $84,4 \%$ del área de estudio, lo que representa una variación de 5,3 mm a 10,8 mm, en términos de evapotranspiración media diaria, en prácticamente toda la cuenca.
\end{abstract}

Palabras clave: Producción agrícola; Equilibrio hídrico; Demanda de agua; Riego; Cambio climático.

\title{
1. Introdução
}

Vários estudos demonstram um aumento na temperatura média da terra causando intensificações dos ciclos hidrológicos, essas mudanças poderão ocasionar variações nos regimes hídricos, como aumento da ocorrência e intensidade dos eventos hidrológicos extremos, modificando a disponibilidade hídrica e a qualidade de vida da população. A análise da tendência de séries históricas de temperatura e precipitação é uma das maneiras de se inferir a ocorrência de mudanças climáticas (Fleury, Miguel \& Taddei, 2019; Silva, Montenegro \& Souza, 2017).

Na bacia hidrográfica do rio Parnaíba no Piauí ocorrem duas estações: uma seca e outra chuvosa. O regime pluvial da região sul do Estado inicia suas chuvas na segunda quinzena do mês de outubro, se intensifica no mês de novembro, estendendo-se até março e tendo como trimestre mais chuvoso os meses: dezembro, janeiro e fevereiro (Medeiros, 2020). Contudo, para o restante dos meses registram-se redução significativa nos índices pluviais, onde se sobressaem agosto e setembro como os mais críticos. Durante a estação chuvosa as precipitações são irregulares e de má distribuição.

Devido às particularidades climáticas, o uso da irrigação é de fundamental aplicabilidade para viabilizar e incrementar a produção agrícola na área da bacia hidrográfica do rio Uruçuí Preto (BHRUP), adquirindo caráter de irrigação suplementar 
na estação chuvosa e com irrigação total na estação seca (Andrade Junior et al., 2018). A evapotranspiração é um elemento climático muito importante para o dimensionamento de sistemas e manejo d'água na irrigação, constituindo-se a sua estimativa mensal no setor regional, de extrema importância nos planejamentos agrícolas (Freitas et al., Costa et al., 2018).

Segundo Wang et al., (2016) a evapotranspiração é regulada pela disponibilidade energética, pelos processos atmosféricos e pelo fornecimento d'água do solo às plantas. A disponibilidade energética depende do local e da época do ano. O local é caracterizado pelas coordenadas geográficas (latitude e longitude) e sua orografia (Horikoshi et al., 2007).

Medeiros et al. (2020) mostraram que a variabilidade climática é um dos elementos mais estudados na dinâmica climática, e os impactos produzidos por esse fenômeno, mesmo estando dentro dos limites esperados, podem ocasionar danos significativos para atividades humanas. Os mesmos autores ainda analisaram a variabilidade climática da umidade relativa do ar e da temperatura máxima do ar na BHRUP, enfocando tais variações como um meio para compreender futuras mudanças. Utilizaram-se dados de temperatura máxima e umidade relativa do ar e totais pluviométricos mensais e anuais no período de 1960 a 1990. Afirmaram que as temperaturas máximas anuais aumentaram durante o período analisado, podendo acarretar vários problemas socioeconômicos, e também para a saúde humana. Verificaram que a umidade relativa do ar está diminuindo ao longo de uma série estudada, fato que pode estar relacionado com o aumento da temperatura. Sobre os totais pluviométricos anuais, nota-se que os valores estão aumentando gradativamente, sendo que esse aumento pode estar relacionado com o aumento da temperatura, que faz com que se tenha uma maior evaporação e consequentemente uma maior precipitação.

Normalmente estimam-se dados de temperatura média do ar, na escala mensal, de determinado local ou região, visando aplicação do método de Thornthwaite (1948), o qual tem sido difundido e utilizado para estimar a Evapotranspiração Potencial (ETP) nas regiões onde não se têm estações meteorológicas.

A transferência d'água de determinada superfície qualquer para a atmosfera, através de processos de evaporação e transpiração é denominada evapotranspiração. A evapotranspiração potencial pode ser definida como sendo a transferência de água para a atmosfera, por evaporação e transpiração, de superfícies vegetadas extensas e uniformes, cobertas por vegetação de porte baixo e bem suprida de água. (Andrade Junior et al., 2018; Alves et al., 2017).

Segundo Marengo, Torres e Alves (2017), a região Nordeste do Brasil (NEB) se caracteriza naturalmente, com alto potencial para evaporação da água em função da grande disponibilidade da energia solar e altas temperaturas. Aumentos de temperatura associados à mudança de clima, decorrente do aquecimento global, independente do que possa vir a ocorrer com as chuvas, já seriam suficientes para causar maior evaporação dos lagos, açudes e reservatórios. Tais aumentos também ocasionam maior demanda evaporativa das plantas, pois a água se tornará um bem mais escasso, com sérias consequências para a sustentabilidade do desenvolvimento regional. A região semiárida do nordeste do Brasil com o passar dos anos, vem se tornando um importante laboratório, possibilitando diversos estudos sobre a precipitação pluviométrica, tendo em vista a sua alta variabilidade temporal e espacial (Júnior \& Bezerra, 2018).

Segundo Aragão (1975) a principal razão da existência do Semiárido nordestino é a ausência de um mecanismo dinâmico que provoque movimentos ascendentes. Secas prolongadas tornam a água um recurso indisponível e até escasso, provocando a migração da população para outras regiões em busca de melhores condições de sobrevivência de vida. A falta de precipitação atrapalha o desenvolvimento agropecuário trazendo consequências negativas para a economia regional. Nas plantas, a falta de água prejudica o seu desenvolvimento em suas diversas fases de crescimento, principalmente, quando este problema ocorre durante fases fenológicas nas quais elas necessitam de maiores quantidades de água, como, por exemplo, durante a floração e frutificação (Fietz et al., 1998).

Outras problemáticas esperadas no Brasil, segundo as projeções dos estudos de mudanças climáticas, são as reduções dos índices pluviométricos que poderão atingir uma faixa de $60 \%$ dos valores mensais. Com isto os reservatórios de armazenamento de águas ficarão obsoletos restringindo ainda mais a água potável para a sobrevivência humana, animal e 
vegetal, como a fauna e a flora, podendo algumas espécies entrar em extinção (Marengo, Torres \& Alves (2017).

Estudos em bacias hidrográficas sobre conflitos de uso de solo e riscos de inundações, possibilitam uma melhor compreensão do meio ambiente, fornecendo o conhecimento necessário para a geração de políticas públicas sobre o uso e conservação de solo e água (Braga et al., 2020, 2021). Pereira et al. (2002) explicam que a BHRUP tem um número limitado de estações ou instrumentos que coletam dados de temperatura do ar e se faz necessário utilizar a estimativa de temperatura tomando como base as coordenadas geográficas locais.

Cavalcanti et al. (2006) desenvolveram equações empíricas para estimar as temperaturas máximas, médias e mínimas do ar para a região Nordeste do Brasil e em especial o Estado do Piauí. Como em todo o Nordeste brasileiro e no território piauiense, as oscilações térmicas dependem mais das variações topográficas do que das decorrentes de variações latitudinais (Sales \& Ramos, 2000).

Com o presente trabalho objetiva-se estimar a evapotranspiração mensal e anual para a área da bacia hidrográfica do rio Uruçuí Preto, tomando-se como modelo as equações de estimativa da temperatura do ar propostas por Cavalcanti et al. $(1994,2006)$.

\section{Metodologia}

A região é drenada pelos afluentes Castros, Ribeirão dos Paulos, Colheres e o Morro da Água e pelos riachos da Estiva e Corrente, ambos rios perenes, e pelo rio Uruçuí Preto. A BHRUP se encontra preponderantemente inserida na bacia sedimentar do rio Parnaíba, sendo um dos principais tributários na margem direita. A BHRUP Possui uma área total aproximada de $15.777 \mathrm{~km}^{2}$, representando $5 \%$ do território piauiense abrangendo parte da região sudoeste, projetando-se em forma de lança, do sul para o norte (COMDEPI, 2002).

A área integral, total ou parcial ocupa 6 folhas na escala 1:250.000 e se situa nas coordenadas geográficas em forma de retângulo de $07^{\circ} 18^{\prime} 16^{\prime \prime}$ a $09^{\circ} 33^{\prime} 06^{\prime \prime}$ de latitude sul e $44^{\circ} 15^{\prime} 30^{\prime \prime}$ a $45^{\circ} 31^{\prime} 11^{\prime \prime}$ de longitude oeste a partir do meridiano de Greenwich (Figura 1).

Segundo o COMDEPI (2002) a BHRUP evidencia um único conjunto de formas de relevo regional, dominadas pelas formas tabulares-planaltos e platôs, característicos das rochas sedimentares sub-horizontalizadas. Apenas o Planalto da Bacia Sedimentar do Parnaíba é identificado como unidade morfoestrutural na região e além de se localizar na porção centro-oriental da Bacia Sedimentar do Piauí-Maranhão é constituída por uma sequência de sedimentos arenoargilosos, compondo as diversas formações sedimentares.

Segundo EMBRAPA (1986) a bacia do rio Uruçuí apresenta três classes frequentes de solos identificadas: Latossolos Amarelos (predominate na bacia), e Neossolos Quatzarênicos e Hidromórficos. 
Figura 1. Localização da bacia hidrográfica do rio Uruçuí Preto no estado do Piauí (escala 1:4.500.000).

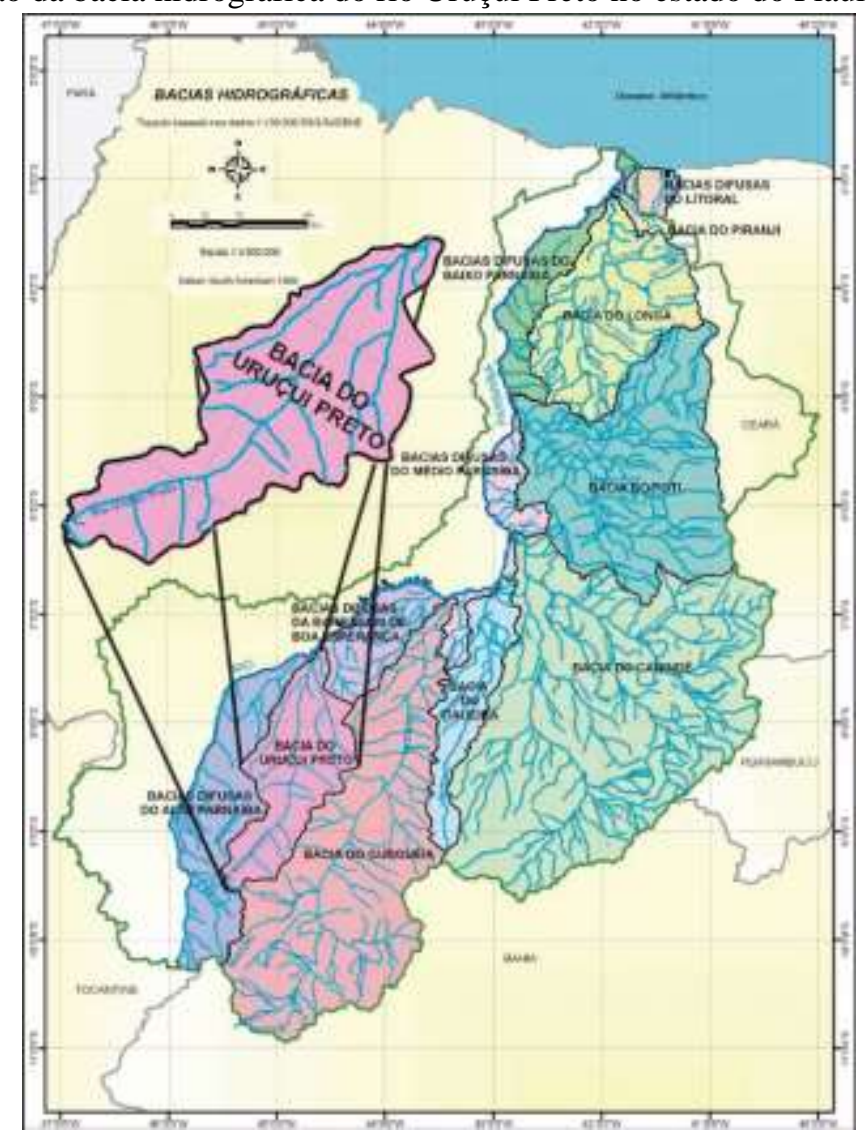

Fonte: COMDEPI (2002).

Conforme a COMDEPI (2002) o fornecimento de água subterrânea na BHRUP ocorre através de 04 (quatro) aquíferos: Serra Grande, Cabeças, Poti/Piauí e Formação Pedra de Fogo. A Formação Serra Grande se estrutura preponderantemente de arenitos grosseiros e médios, conglomeráticos e conglomerados em vários níveis (creme a branco) com estratificação cruzada plana. Embora se configure como um dos mais destacados do Nordeste, inclusive se distribuindo por toda a Bacia Sedimentar do Parnaíba, não oferece possibilidades eficientes de exploração na região da bacia do rio Uruçuí Preto, em razão das grandes profundidades.

Segundo COMDEPI (2002) a identificação e descrição da vegetação na região da BHRUP ocorre a partir do topo das chapadas, com a comunidade vegetal típica das savanas constituída de um estrato descontínuo composto de elementos arbustivos e arbóreos, caracterizados por troncos tortuosos, casca espessa, folhas coriáceas e dossel quase sempre assimétrico. Entre as espécies mais frequentes estão o barbatimão, o pau terra de folha larga e a simbaíba. A superfície do solo é recoberta por um estrato graminoso de capim agreste, a começar das vertentes entre o topo das chapadas e o trecho plano por onde corre o rio Uruçuí. Nesta vertente o cerrado se desenvolve de forma mais fechada, composto por espécies de maior porte, entre as quais o pau d'arco e o Gonçalo Alves.

A bacia é composta de regiões assoladas por eventos sinóticos locais, regionais por mecanismos de grandes escalas proporcionando eventos de secas, enchentes, inundações, alagamentos e transbordamento de rios, açudes, lagoas, lagos e córregos. Na sua maioria, o escoamento dos rios nas cabeceiras é temporário devido à má distribuição dos índices pluviométricos na região piauiense. O período chuvoso com os aumentos das suas cotas pluviométricas provoca um aumento significativo no escoamento, em que a maioria é represada em grandes e médias barragens e seu excesso, após os represamentos, escoa lentamente para o oceano em virtude do relevo e de seus cursos básico das águas.

As enchentes e as inundações já provocaram prejuízos e remoções de diversos povoados e vilas; historicamente as 
maiores cheias ocorreram entre os trechos dos médio e baixo Parnaíba, na BHRUP. A ocorrência de enchentes é quase que periódica, dependo da qualidade do período chuvoso, pois, nesta área não existem sistemas de contenção de enchentes e suas vazões são aleatórias auxiliadas pelo relevo (SUDENE, 1999).

As chuvas na área da BHRUP ocorrem nos meses de outubro a março, com o trimestre mais chuvoso entre meses de dezembro, janeiro e fevereiro, representando em torno de $65 \%$ dos índices anuais de chuvas, quando em épocas de período de normalidade. O período chuvoso coincide com a época das penetrações ou das atividades das frentes frias mais intensas, que atingem a área estudada. Outro fator que contribui para as atividades das chuvas são as formações dos Vórtices Ciclônicos do Atlântico Sul (VCAS), quando do seu posicionamento adequado e o auxílio das contribuições locais e regionais (Medeiros, 2013).

Alguns aspectos geográficos conferem ao Piauí, excelentes condições de potencialidade hídrica. Está situado na bacia sedimentar do rio Parnaíba, que conta com caráter perene, e uma extensão de $1.485 \mathrm{~km}$, com uma vazão média da ordem de $600 \mathrm{~m}^{3} \mathrm{~s}^{-1}$, sendo o menor fluxo em torno de $280 \mathrm{~m}^{3} \mathrm{~s}^{-1}$ no seu trecho superior. Pelo seu leito são drenados cerca de 20 bilhões de $\mathrm{m}^{3} \mathrm{ano}^{-1}$.

Os postos fluviométricos existentes em Barra do Lance, município de Jerumenha, e Cristino Castro, mostram vazões médias de $6,9 \mathrm{~m}^{3} \mathrm{~s}^{-1}$ e $6,1 \mathrm{~m}^{3} \mathrm{~s}^{-1}$ no trimestre mais seco e vazões médias, respectivamente, de $90,0 \mathrm{~m}^{3} \mathrm{~s}^{-1}$ e $54,0 \mathrm{~m}^{3} \mathrm{~s}^{-1}$, no trimestre mais chuvoso.

Parte relativamente pequena da bacia, é constituída de terrenos cristalinos, entretanto, na maior parte da área afloram sedimentos da bacia sedimentar do Parnaíba.

As atividades antrópicas quase sempre modificam os aspectos naturais presentes nos territórios nas quais são desenvolvidas, como a pedologia, a geomorfologia e a vegetação. O meio ambiente de alguma forma se encontra inserido no contexto de uma bacia hidrográfica, que pode vir a sofrer drásticas transformações e modificações no tocante à sua dinâmica natural, criando assim, ambientes artificiais. As formas de relevo fluviais são elaboradas por meio do escoamento concentrado de água em canais fluviais. Esse escoamento, cujo volume depende do regime hidrológico da bacia hidrográfica, funciona como um dos agentes modeladores do ambiente onde essa bacia está inserida, sua alteração corrobora para a criação de uma nova forma de estruturação do ambiente que, se não receber adequações para sua utilização, pode agir como potencializador do risco, gerando então perdas econômicas e de vidas humanas, vegetais e animais.

A estimativa do poder evapotranspirativo mínimo, máximo e anual efetuou-se a partir das equações empíricas para a estimativa da temperatura para a área da BHRUP, conforme Medeiros (2013). O autor utilizou séries históricas mensais da temperatura uniformizados com 30 anos de observações, para 49 municípios que engloba a área de estudo. Com os dados foram ajustadas equações pelo método dos mínimos quadrados, apresentado a seguir:

$$
\mathrm{E}(\mathrm{Y})=\mathrm{a}_{0}+\mathrm{a}_{1} \mathrm{X}_{1}+\mathrm{a}_{2} \mathrm{X}_{2}+\mathrm{a}_{3} \mathrm{X}_{3}
$$

Onde:

Y - temperatura mensal do ar (média, máxima ou mínima) ${ }^{\circ} \mathrm{C}$;

$\mathrm{X}_{1}, \mathrm{X}_{2}$ e $\mathrm{X}_{3}$ - latitude e longitude do local (minuto) e altitude (metro), respectivamente; $a_{0}, a_{1}, a_{2}$ e $a_{3}$ - coeficientes da equação de regressão.

As equações de regressão linear múltipla para estimar a temperatura (máxima, média e mínima) foram obtidas com base na latitude, longitude e altitude locais assim como os dados pluviais referentes ao mesmo período de estudo. Os dados das coordenadas geográficas foram utilizados do banco de dados do Instituto Brasileiro de Geografia e Estatística - IBGE e com os dados de temperatura média estimados, procedeu-se à estimativa da evapotranspiração, pelo método empírico de Thornthwaite (1948), Thornthwaite e Mather (1955).

Com o intuito de homogeneizar as classes da evapotranspiração, quando em determinado município ocorressem duas 
ou mais classes de evaporação potencial, estabeleceram-se duas situações: Mudança de classe inferior para classe superior quando a porcentagem de ocorrência de uma classe inferior à de outra classe fosse igual a 20\%, a área de ocorrência da classe inferior seria incorporada à da classe imediatamente superior; Mudança de classe superior para classe inferior - quando a porcentagem de ocorrência de uma classe superior à de outra classe fosse igual a 20\%, a área de ocorrência da classe superior seria incorporada à da classe imediatamente inferior. Este procedimento possibilitou a obtenção de mapas de evapotranspiração mais uniformes e homogêneos.

Foram elaborados valores da temperatura média do ar, estimados pelo software Estima_T (Cavalcanti e Silva, 1994; Cavalcanti e Silva, 2006) estando disponível no site da Unidade Acadêmica de Ciências Atmosféricas da Universidade Federal de Campina Grande.

O modelo empírico de estimativa da temperatura do ar é uma superfície quadrática para a temperatura média mensal, em função das coordenadas locais: longitude, latitude e altitude (Cavalcanti e Silva, 2006), sendo dada por:

$$
\mathrm{T}=\mathrm{C} 0+\mathrm{C} 1 \lambda+\mathrm{C} 2 \varnothing+\mathrm{C} 3 \mathrm{~h}+\mathrm{C} 4 \lambda 2+\mathrm{C} 5 \varnothing 2+\mathrm{C} 6 \mathrm{~h} 2+\mathrm{C} 7 \lambda \varnothing+\mathrm{C} 8 \lambda \mathrm{h}+\mathrm{C} 9 \varnothing \mathrm{h}
$$

Onde:

$\mathrm{C} 0, \mathrm{C} 1, \ldots . ., \mathrm{C} 9$ são as constantes; $\lambda, \lambda 2, \lambda \varnothing, \lambda \mathrm{h}$ longitude; $\varnothing, \varnothing 2, \lambda \varnothing$ latitude; $\mathrm{h}, \mathrm{h} 2, \lambda \mathrm{h}, \varnothing \mathrm{h}$ altura.

Também se pode estimar a série temporal de temperatura, adicionando-se a esta, a anomalia de temperatura do Oceano Atlântico Tropical (Cavalcanti e Silva, 2006).

$$
\mathrm{Tij}=\mathrm{Ti}+\mathrm{AATij}
$$

Onde:

$i=1,2,3, \ldots, 12 \quad j=1950,1951,1952,1953, \ldots, 2014$.

Foi elaborada planilha eletrônica com os dados obtidos com o Estima_T, e calculada a média anual da temperatura média do ar. Utilizando-se o software Surfer 9.0, foi elaborada a estatística utilizando método da krigagem elaborando-se o mapa anual, utilizando-se o limite do Estado da Paraíba (IBGE, 2011).

\section{Resultados e Discussão}

A oscilação da ETP de referência média da área estudada ocorre entre 92,4 mm, em fevereiro, e 170,1 mm no mês de outubro. A ETP máxima oscila entre 111,3 e 202,8 mm; já a ETP mínima oscila entre 70,7 e 130,2 mm.

Comparando-se os índices evapotranspirados com os precipitados, conclui-se ser um número significativo de água evapotranspirada em relação aos índices precipitados. Os valores estudados da evapotranspiração são representativos da média dos 25 postos pluviométricos e das 24 fazendas, em que se trabalhou com o valor médio, valores máximos e mínimos (Figura 2).

Gomes et al. (2005) estimaram a evapotranspiração mensal para o estado do Piauí, pelo método empírico de Thornthwaite (1948), com base nas equações de estimativa da temperatura média do ar, propostas por Medeiros (2016) Estimaram-se a ETP para os 222 municípios do Estado, cujos valores foram geoespacializados e agrupados em classes de ETP, com intervalo de $25 \mathrm{~mm}$, gerando-se mapas de ETP mensais. Os valores de ETP mensais variaram de 55,4 a 212,0 mm, evidenciando a variação na demanda evapotranspirativa das diversas regiões do estado. O período de fevereiro a julho apresentou os menores valores mensais de ETP, enquanto de setembro a dezembro, os maiores valores. Esta tendência é um reflexo da variação espacial da temperatura média do ar mensal nas diferentes regiões do estado, sinalizando que esses resultados estão em conformidade com os apresentados no estudo da BHRUP. 
Figura 2. Representação da Evapotranspiração média das máximas; média das médias e médias das mínimas.

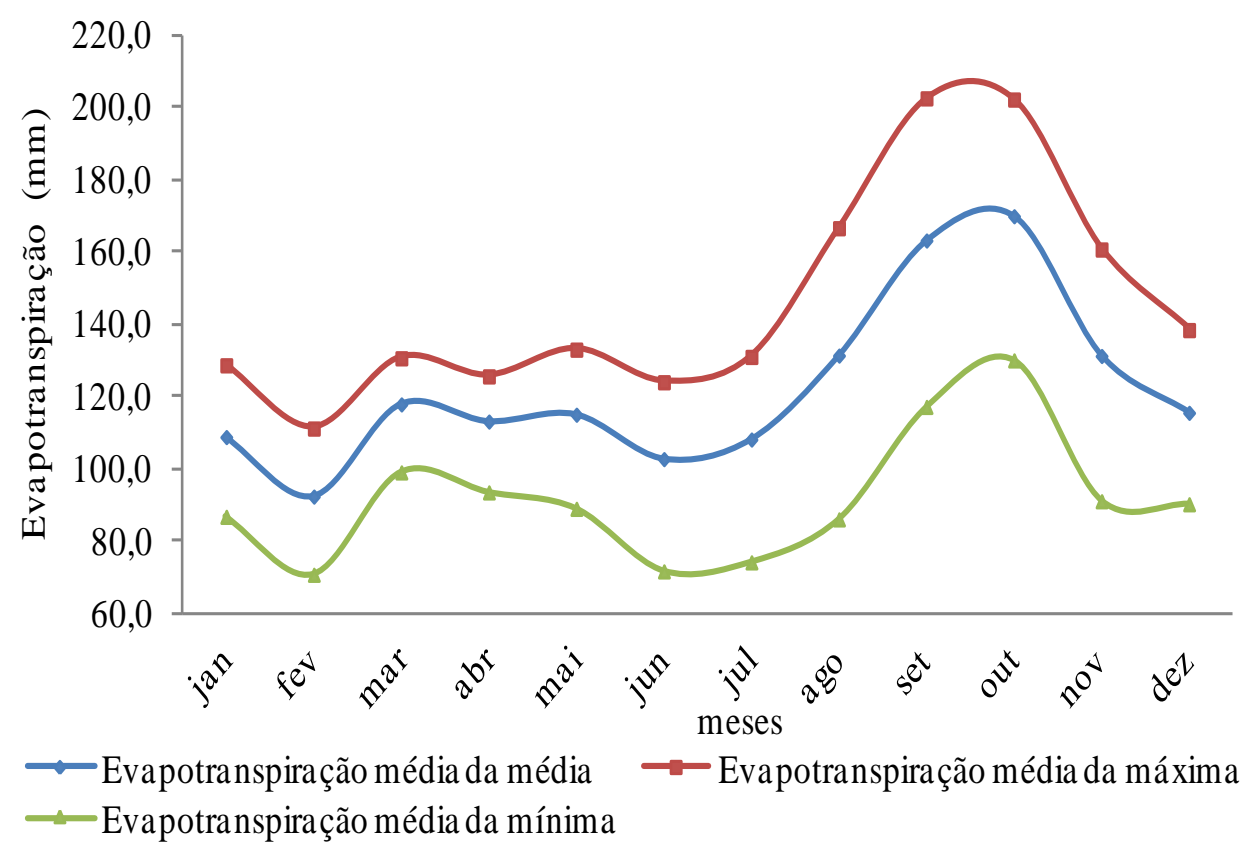

Fonte: Medeiros (2016).

A Figura 3 (a, b, c) representa a variabilidade da evapotranspiração (a) mínima, (b) máxima e (c) anual, para a área da BHRUP.

Figura 3. Evapotranspiração (mm): (a) mínima; (b) máxima; (c) anual.
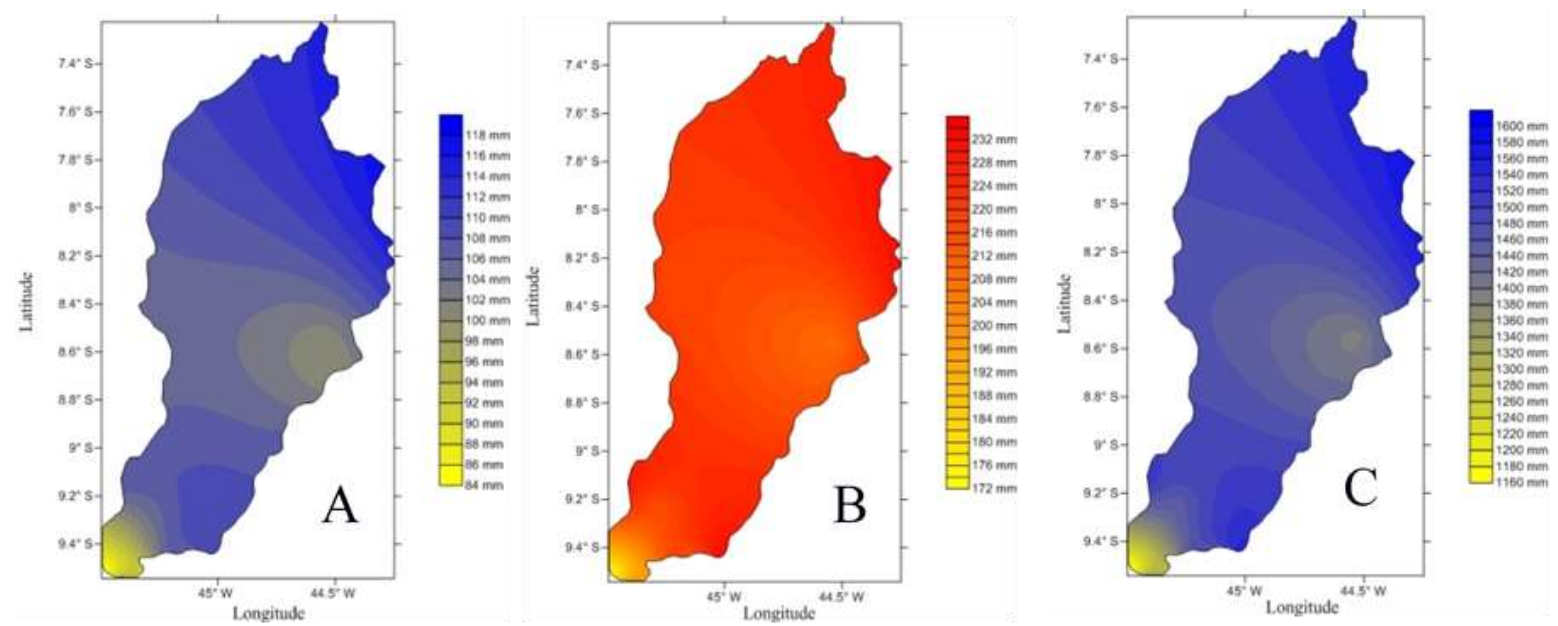

Fonte: Medeiros (2016).

Na Figura 3a se encontram os índices de evapotranspiração mínima que ocorrem no mês de fevereiro, com flutuação de 84 a $118 \mathrm{~mm}$. As áreas com menores índices de ETP estão localizadas no extremo sul e na parte leste da região central, enquanto nos setores norte, nordeste e sudeste, se localizam os altos índices de ETP.

Na Figura $3 b$ se observa acréscimo de sul-norte, em toda a BHRUP, sendo que valores mínimos dos máximos se localizam no extremo sul e em uma pequena faixa ao leste da área central.

Na Figura 3c se observam as menores taxas de evapotranspiração anual no extremo sul e na área leste da região central. As demais áreas se apresentam com evapotranspirações em elevações, cujos valores máximos ocorrem nos setores norte, nordeste e sudoeste. A flutuação anual ocorre na faixa de 1.160 a $1.600 \mathrm{~mm}$. 


\section{Conclusão}

As estimativas de ETP mensais para a área da BHRUP apresentam dependência da localização geográfica (latitude e longitude) e da topografia local (altitude).

Em termos médios, predominam as classes de ocorrência de 70 a 125 mm; 125 a 175 mm e a classe mais elevada acima dos $200 \mathrm{~mm}$ que, juntas, totalizam 84,4 \% da área de estudo, representando uma variação de 5,3 a 10,8 mm, em relação a ETP média diária, em praticamente toda a área de estudo.

Quanto à evapotranspiração real, quantificada em relação à precipitação média, as regiões de Parnaíba, Atlântico Nordeste Oriental, Atlântico Leste, São Francisco, Paraguai e Atlântico Nordeste Ocidental, apresentaram índices de precipitação entre $94 \%$ e $83 \%$. Valores maiores que $80 \%$ da precipitação média, ocasionaram menores percentuais de escoamento superficial efetivo.

\section{Referências}

Alves, W. B., Rolim, G. S., \& Aparecido, L. E. O. (2017). Reference evapotranspiration forecasting by artificial neural networks. Agricultural Engineering, 37(6), 1116-1125.

Andrade Junior, A. S., Silva, C. O., Sousa, V. F., \& Ribeiro, V. Q. (2018). Evaluation of methods for estimating reference evapotranspiration in the state of Piauí. Agrometeoros, 25(1), 181-190.

Aragão, J. O. R. (1975). Um estudo das estruturas das perturbações sinóticas do Nordeste do Brasil. (INPE-789-TPT/017). Dissertação (Mestrado em Meteorologia).

Braga, S. E., Caldas, A. M., Rizzi Neto, E., Silva, L. J. DE S., Perônico, A. M. B. L., Wanderley, R. A., Freitas, P. M. L. de, Lima, J. R. de, Cunha, C. R. R. O. da, \& Abreu, B. S. (2021). Environmental conflict of land use in Tapacurá-PE hydrographic basin. Research, Society and Development, $10(2)$, e52310212833. https://doi.org/10.33448/rsd-v10i2.12833

Braga, S. E., Wanderley, R. A., Holanda, T. F., \& Caldas, A. M. (2020). Aplicação da Análise Hierárquica para mapeamento de risco de inundação: O caso no município de Limoeiro (Pernambuco - Brasil). Meio Ambiente (Brasil), 2(1), 57-66. https://doi.org/10.5281/zenodo.3969577

Cavalcanti, E. P., \& Silva, E. D. V. (1994). Estimativa da temperatura do ar em função das coordenadas locais. IN: Congresso Brasileiro de Meteorologia. 8. 1994. Belo Horizonte, Anais... Belo Horizonte: SBMET, 1, 154-157pp.

Cavalcanti, E. P., Silva, V. P. R., \& Sousa, F. A. S. (2006). Programa computacional para a estimativa da temperatura do ar para a região Nordeste do Brasil. Revista Brasileira de Engenharia Agrícola e Ambiental, Brasil, 10(1), 140-147.

COMDEPI. Companhia de desenvolvimento do Piauí. (2002). Estudo de viabilidade para aproveitamento hidroagrícola do vale do rio Uruçuí Preto. Teresina.

Costa, J. A., Rodrigues, G. P., Silva, N. D., Nephew, O. P. L., \& Costa, L. D. (2018). Evaluation of methods for estimating reference evapotranspiration for Alagoas. Agrometeoros, 25(1), 24-36.

EMBRAPA. Empresa Brasileira de Pesquisas Agropecuárias (1986). Levantamento exploratório-reconhecimento de solos do Estado do Piauí. Vol. SNLCS.

Fietz, C. R., Frizzone, F. A., \& Folegatti, M. V. (1998). Probabilidade de ocorrência de períodos secos e chuvosos na região de Dourados, MS. Irriga (Botucatu), Botucatu, 3(1), 16-22.

Fleury, L. C., Miguel, J. C. H., \& Taddei, R. (2019). Mudanças climáticas, ciência e sociedade. Sociologias, 21(51), 18-42.

Florenzano, T. G. (2008). Os satélites e suas aplicações.

Freitas, P. A., Oliveira, L. M. M., Sales Barbosa, R. F., \& Montenegro, S. M. G. (2018). Evapotranspiration of daily reference by different models in the capibaribe river basin (PernambucoBrazil). Revista Brasileira de Ambiente, 4(1), 25- 31.

Gomes, A. A. N., Andrade Júnior, A. S., \& Medeiros, R. M. (2005). Evapotranspiração de referência mensal para o Estado do Piauí. Revista Brasileira de Engenharia Agrícola e Ambiental Campina Grande, PB. 9(4), 560-564.

Horikoshi, A. S., \& Fisch, G. (2007). Balanço Hídrico Atual e Simulações para Cenários Climáticos Futuros no Município de Taubaté, SP, Brasil. Revista Ambiente e Água. An Interdisciplinary Journal of Applied Science, 2(2).

IBGE. (2011). Instituto Brasileiro de Geografia e Estatística. http://www.ibge.gov.br.

Júnior, J. B. C., \& Bezerra, B. G. (2018). Análises da evapotranspiração de referência e do índice de aridez para o Nordeste do Brasil. Revista de Geociências do Nordeste, 4(1), 71-89.

Marengo, J. A., Torres, R. R., \& Alves, L. M. (2017). Drought in Northeast Brazil—past, present, and future. Theoretical and Applied Climatology, 129(3-4), $1189-1200$ 
Research, Society and Development, v. 10, n. 5, e42810514752, 2021

(CC BY 4.0) | ISSN 2525-3409 | DOI: http://dx.doi.org/10.33448/rsd-v10i5.14752

Medeiros, R, M., Sousa, F, A. S., Gomes Filho, M. F., \& Paulo R. M. (2013). Variabilidade da umidade relativa do ar e da temperatura máxima na bacia hidrográfica do rio Uruçuí PrETP. Revista Educação Agrícola Superior. Associação Brasileira de Educação Agrícola Superior - ABEAS - 28(1), p.xx-xx. http://dx.doi.org/10. 12722/0101-756X.v28n01axx

Medeiros, R. M. (2013). Estudo agrometeorológico para o Estado do Piauí. P.114.

Medeiros, R.M, Cunha Filho, M., Cunha, A. L. X., Holanda, R. M., Franca, M. V., Araujo Filho, R. N., Piscoya, V. C., Moreira, G. R., Nascimento, G. I. L. A., \& Gomes Filho, R. R. (2020) Climate Variables of the Uruçuí Preto-Piauí Hydrographic Basin, Brazil. ijsrm.human, 15 (3), 124-142.

Ortolani, A. A., \& Camargo, M. B. P. (1987). Influência dos fatores climáticos na produção. In: Castro, P.R.C., Ferreira, S.O., Yamada, T. Ecofisiologia da produção agrícola. Piracicaba: Associação Brasileira para Pesquisa da Potássio e do Fosfato, p.71-81.

Pereira, A. R., Angelocci, L. R., \& Sentelhas, P. C. (2002). Agrometeorologia: Fundamentos e aplicações práticas. Guaíba: Agropecuária, p.105-129.

Sales, M. C. L., \& Ramos, V. M. (2000). Caracterização ambiental das áreas sob influência do reservatório de Bocaina (PI) com base na compartimentação geomorfológica. In: Carta CEPRO, Teresina, 18(1), 149-161.

Silva, R. O. B. D., Montenegro, S. M. G. L., \& Souza, W. M. D. (2017). Tendências de mudanças climáticas na precipitação pluviométrica nas bacias hidrográficas do estado de Pernambuco. Engenharia Sanitaria e Ambiental, 22(3), 579-589.

SUDENE - Superintendência de Desenvolvimento do Nordeste. (1999). Dados pluviométricos mensais do Nordeste - Paraíba. Recife, (Série Pluviometria, 2).

Thornthwaite, C. W., \& Mather, J. R. (1955). The water balance. Publications in Climatology. New Jersey: Drexel Institute of Technology, 104p.

Thornthwaite, C. W. (1948). An approach toward a rational classification of climate. Geogr. Rev, 38, 55-94.

Varejão Silva, M. A. (2000) Meteorologia e Climatologia. Brasília: INMET, Gráfica e Editora STILO. 532 p.: il.

Wang, W., Ertsen, M. W., Svoboda, M. D., \& Hafeez, M. (2016). Propagation of drought: From meteorological drought to agricultural and hydrological drought. Advances in Meteorology. 